\title{
Valores hematológicos de bugios pretos (Alouatta caraya) de vida livre da região do Alto Rio Paraná, sul do Brasil
}

[Hematologic values of free-ranging black-and-gold howlers monkeys (Alouatta caraya) from the region of Upper Paraná River, Southern Brazil]

\author{
K.K.M.C. Flaiban ${ }^{1}$, K.A.H. Spohr ${ }^{1}$, L.S. Malanski ${ }^{2}$, W.K. Svoboda ${ }^{3}$, M.M. Shiozawa ${ }^{1}$, C.L.S. Hilst ${ }^{1}$, \\ L.M. Aguiar ${ }^{3}$, G. Ludwig ${ }^{3}$, F.C. Passos ${ }^{3}$, I.T. Navarro ${ }^{1}$, J.A.N. Lisbôa ${ }^{1}$, M.R.S. Balarin ${ }^{1}$ \\ ${ }^{1}$ Universidade Estadual de Londrina - Londrina, PR \\ ${ }^{2}$ IBAMA - Curitiba, PR \\ ${ }^{3}$ Universidade Federal do Paraná - Curitiba, PR
}

\begin{abstract}
RESUMO
Descreveram-se os valores hematológicos de bugios pretos (Alouatta caraya) e estabeleceram-se diferenças entre sexo e idade, de 36 animais aparentemente saudáveis, de vida livre da região do Alto Rio Paraná, sul do Brasil. Os animais foram capturados e contidos quimicamente com cloridrato de tiletamina e cloridrato de zolazepam, na dose média de $5,5 \mathrm{mg} / \mathrm{kg}$, por via intramuscular. Na comparação entre os sexos, houve diferença para número de hemácias $\left(4,48 \pm 1,36\right.$ e $\left.3,58 \pm 0,91 \times 10^{6} / \mathrm{mm}^{3}\right)$, maior nas fêmeas, e volume globular médio $(90,99 \pm 27,65$ e 118,36 $\pm 44,63 \mathrm{fL})$ e número de eosinófilos $(0,30 \pm 0,24$ e $0,76 \pm 0,85$ $\left.\mathrm{x} 10^{3} / \mathrm{mm}^{3}\right)$, maior nos machos. O volume globular $(39,46 \pm 3,53$ e $36,69 \pm 3,54 \%)$ e a proteína total plasmática $(7,91 \pm 0,53$ e 7,40 $\pm 0,63 \mathrm{~g} / \mathrm{dL})$ foram significativamente mais baixos nos animais jovens.
\end{abstract}

Palavras-chave: bugio preto, vida livre, hematologia

\begin{abstract}
The hematologic values and the influence of gender and age were described in 36 free-ranging healthy black-and-gold howler monkeys (Alouatta caraya) from the region of Paraná river, Southern Brazil. The animals were caught with trap models and intramuscularly anesthetized with $5.5 \mathrm{mg} / \mathrm{kg}$

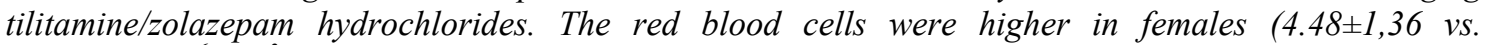
$\left.3.58 \pm 0.91 \times 10^{6} / \mathrm{mm}^{3}\right)$ while mean corpuscular volume $(90.99 \pm 27.65$ and $118.36 \pm 44.63 \mathrm{fL}$ ) and eosinophils $\left(0.30 \pm 0.24\right.$ and $\left.0.76 \pm 0.85 \times 10^{3} / \mathrm{mm}^{3}\right)$ were significant higher in males. The packed cell volume $(39.46 \pm 3.53$ and $36.69 \pm 3.54 \%)$ and plasma total protein $(7.91 \pm 0.53$ and $7.40 \pm 0.63 \mathrm{~g} / \mathrm{dL})$ were lower in juveniles.
\end{abstract}

Keywords: black-and-gold howler monkey, free-ranging, hematology

\section{INTRODUÇÃO}

Os exames hematológicos são de grande auxílio ao clínico veterinário para estabelecer o diagnóstico, estimar a gravidade da doença e, em alguns casos, determinar o prognóstico, além de monitorar a resposta à terapia (Barger, 2003). O conhecimento dos intervalos fisiológicos dos valores hematológicos torna-se necessário para auxiliar na compreensão das alterações provocadas por agentes patogênicos, estabelecendo os limites entre o estado de saúde e o de doença (Moore, 2000).

Os primatas do gênero Alouatta têm ampla distribuição geográfica, ocorrendo do México ao sul do Brasil e norte da Argentina. Também são amplamente estudados em projetos de translocação e reintrodução e nos aplicados ao comportamento (Silveira e Codenotti, 2001; 
Thoisy et al., 2001; Aguiar et al., 2003). Entretanto, valores hematológicos para primatas deste gênero foram apontados apenas para $A$. villosa (Porter Junior, 1971), A. seniculus (Vie et al., 1998) e A. caraya (Satake, 2006). Perfis fisiológicos de primatas neotropicais raramente são estabelecidos, pois esses animais são difíceis de capturar, e as condições de campo, muitas vezes, são desfavoráveis (Vie et al., 1998). Estes animais podem ser reservatórios silvestres de importantes doenças como febre amarela (Hull et al., 1991), malária (Thoisy et al., 2000) e amebiose (Thoisy et al., 2001), dentre outras.

De maneira geral, os constituintes do eritrograma de primatas variam com o sexo e a idade. Os neonatos possuem número de hemácias, volume globular e concentração de hemoglobina maiores do que os animais adultos (Thrall, 2004). Os valores nos machos adultos são maiores que em fêmeas adultas (Vie et al., 1998; Larsson et al., 1999; Moore, 2000; Riviello e Wirz, 2001), mas as variáveis do leucograma podem sofrer influência apenas da idade (Vie et al., 1998; Riviello e Wirz, 2001). Excitação e estresse ligados à captura podem produzir contração esplênica e aumento do volume globular (Thrall, 2004). Quanto ao leucograma, o estresse pode provocar elevações do número de leucócitos e de neutrófilos segmentados (Cunha et al., 2005).

Em decorrência da escassez de literatura sobre o assunto, os objetivos deste trabalho foram descrever os valores hematológicos em bugios pretos (Alouatta caraya) de vida livre, da região do Alto Rio Paraná, sul do Brasil, e estabelecer possíveis diferenças entre o sexo e a idade.

\section{MATERIAL E MÉTODOS}

Foram colhidas amostras de sangue de 36 primatas, da espécie Alouatta caraya, aparentemente saudáveis, de vida livre da região do Alto Rio Paraná, sul do Brasil, no período de julho de 2004 a dezembro de 2005.

A área de estudo localiza-se na região do município de Porto Rico, noroeste do estado do

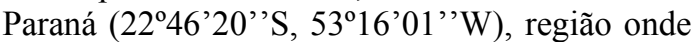
o Rio Paraná apresenta um complexo e dinâmico sistema de arquipélagos fluviais. Nesta área, as matas ciliares foram quase totalmente devastadas a partir da década de 1960. Atualmente, a maioria da formação vegetacional arbórea da região encontra-se em processo de regeneração, formando um misto de fragmentos não perturbados e áreas de vegetação mais aberta e alterada (Aguiar, 2006).

A captura e a manipulação dos primatas foram autorizadas pelo Instituto Brasileiro do Meio Ambiente e Recursos Naturais Renováveis (IBAMA). O projeto foi aprovado para execução pelo Comitê de Ética em Experimentação Animal da Universidade Estadual de Londrina (CEEA/UEL).

Os animais foram capturados por meio de armadilhas manuais ou automáticas, conforme descrito por Aguiar et al. (2006). As armadilhas para a captura dos bugios pretos foram posicionadas nas copas das árvores. Quando ocorria a captura, os animais eram anestesiados e transportados até o solo para a realização dos procedimentos. Após a captura, foram contidos quimicamente com cloridrato de tiletamina e cloridrato de zolazepam ${ }^{1}$, na dose média de $5,5 \mathrm{mg} / \mathrm{kg}$, por via intramuscular (Hilst et al., 2006). Após a anestesia, foram transportados e mantidos em gaiolas de contenção até a realização de procedimentos como marcação individual com transponder (implante subcutâneo) na região interescapular. Os exames físicos constaram de: aferição da temperatura retal e das frequências cardíaca e respiratória, avaliação da coloração das mucosas, do tempo de preenchimento capilar e do pulso, inspeção da pele e pelos, palpação abdominal e mensuração de dados biométricos (Svoboda et al., 2006). Com base nas evidências do exame físico, foram excluídos do estudo os animais julgados não saudáveis. A colheita de sangue foi realizada por venopunção da jugular externa. $O$ volume de sangue destinado para a análise hematológica foi acondicionado em frascos contendo EDTA 5\% como anticoagulante. Após a recuperação da tranquilização, os animais foram transportados até o exato local da captura e postos em liberdade.

Os grupos etários foram classificados por meio do tamanho corporal, do desenvolvimento das características sexuais, da coloração da pelagem e da avaliação dos dentes (tamanho, coloração, desgaste), conforme preconizado pelo Centro

${ }^{1}$ Zoletil $^{\circledR}$ - Virbac - São Paulo, Brasil.rs 
Nacional de Primatas, em Belém, Pará. Os primatas idosos e muito jovens (fase infantil) não foram incluídos neste estudo. Os animais foram agrupados ora de acordo com o sexo (16 fêmeas e 20 machos), ora conforme a idade (23 adultos e 13 jovens), para efeitos de comparação.

Realizaram-se análises por meio dos métodos hematológicos tradicionais, segundo Jain (1993), compreendendo: contagem total de hemácias e de leucócitos realizadas em hemocitômetro manual; determinação do volume globular (VG), por meio da técnica de microematócrito; mensuração da concentração de hemoglobina $(\mathrm{Hb})$ por meio da técnica de cianometaemoglobina com leitura espectrofométrica; cálculo dos índices hematimétricos: volume globular médio (VGM), hemoglobina globular média (HGM) e concentração de hemoglobina globular média
(CHGM); contagem diferencial de leucócitos em extensões sanguíneas coradas pelos corantes de May-Grunwald e Giemsa, e mensuração da concentração da proteína total plasmática (PTP), por meio da refratometria (Coles, 1984). Utilizou-se o teste $\mathrm{t}$ para comparar sexos e idade (adulto e jovem) admitindo-se uma probabilidade de erro de 5\% (Curi, 1997).

\section{RESULTADOS E DISCUSSÃO}

Houve diferenças entre sexos para o número de hemácias, para o VGM e para o número de eosinófilos (Tab. 1) e não foram encontrados bastonetes circulantes nesta espécie de primata. Observou-se coeficiente de variação muito elevado para as contagens de basófilos e de monócitos. Os valores médios dos grupos ficaram entre 20 e 80 basófilos $/ \mu \mathrm{L}$ e 40 e 70 monócitos $/ \mu \mathrm{L}$.

Tabela 1. Média e desvio-padrão de variáveis hematológicas e da concentração de proteína total plasmática de fêmeas e machos da espécie Alouatta caraya de vida livre, da região do Alto Rio Paraná, sul do Brasil, 2007

\begin{tabular}{lccc}
\hline Variável & Fêmea $(\mathrm{n}=16)$ & Macho $(\mathrm{n}=20)$ & $\mathrm{P}$ \\
\hline Hemácias $\left(\times 10^{6} / \mu \mathrm{L}\right)$ & $4,48 \pm 1,36$ & $3,58 \pm 0,91$ & $<0,05$ \\
Hemoglobina $(\mathrm{g} / \mathrm{dL})$ & $11,18 \pm 3,82$ & $9,96 \pm 2,77$ & $\mathrm{~ns}$ \\
Volume globular $(\%)$ & $37,78 \pm 4,03$ & $39,00 \pm 3,49$ & $\mathrm{~ns}$ \\
VGM (fL) & $90,99 \pm 27,65$ & $118,36 \pm 44,63$ & $<0,05$ \\
HGM (pg) & $25,58 \pm 7,65$ & $30,06 \pm 12,66$ & $\mathrm{~ns}$ \\
CHGM $(\%)$ & $29,40 \pm 9,03$ & $25,42 \pm 6,34$ & $\mathrm{~ns}$ \\
Leucócitos $\left(\times 10^{3} / \mu \mathrm{L}\right)$ & $10,18 \pm 6,47$ & $12,17 \pm 7,70$ & $\mathrm{~ns}$ \\
Segmentados $\left(\times 10^{3} / \mu \mathrm{L}\right)$ & $6,98 \pm 5,92$ & $7,43 \pm 5,84$ & $\mathrm{~ns}$ \\
Eosinófilos $\left(\times 10^{3} / \mu \mathrm{L}\right)$ & $0,30 \pm 0,24$ & $0,76 \pm 0,85$ & $<0,05$ \\
Linfócitos $\left(\times 10^{3} / \mu \mathrm{L}\right)$ & $2,83 \pm 1,43$ & $3,82 \pm 1,97$ & $\mathrm{~ns}$ \\
PTP $(\mathrm{g} / \mathrm{dL})$ & $7,91 \pm 0,54$ & $7,57 \pm 0,64$ & $\mathrm{~ns}$ \\
\hline
\end{tabular}

VGM: volume globular médio; HGM: hemoglobina globular média; CHGM: concentração de hemoglobina globular média; PTP: proteínas totais plasmáticas.

O número de hemácias foi mais alto nas fêmeas e o VGM mais elevado nos machos. Estes resultados diferem, de forma geral, de outros obtidos da literatura, que afirmam que os machos possuem valores mais elevados do que as fêmeas (Vie et al., 1998; Larsson et al., 1999; Moore, 2000; Riviello e Wirz, 2001). Com respeito ao diâmetro das hemácias, pode-se considerar a possibilidade de existirem fêmeas no início da gestação, quando o tamanho da hemácia está diminuído (Harewood et al., 2000). A manutenção do valor baixo do VGM na fase final da gestação em mulheres está relacionada à deficiência de ferro (De Leeuw e Lowestein,
1966). No grupo estudado, observaram-se apenas duas fêmeas com filhote, e não houve relato de animais em gestação.

O número de eosinófilos foi mais elevado nos machos, o que não foi observado por outros autores para $A$. villosa (Porter Junior, 1971), $A$. seniculus (Vie et al., 1998) e $A$. caraya (Satake, 2006). Entretanto, independentemente do sexo, primatas, em geral, possuem valores de eosinófilos que podem responder por até $8 \%$ do total de leucócitos (Moore, 2000). 
A dieta dos bugios é baseada no consumo de brotos, folhas e frutos, por isso, esses animais adotam um comportamento que visa poupar energia, com pouca atividade diária (Azevedo e Bicca-Marques, 2005). Em um estudo com $A$. guariba clamitans, não foram observadas diferenças no tempo dedicado à alimentação e na contribuição dos itens alimentares (folha, fruto e flor) para a dieta de animais em diversas faixas etárias dos dois sexos. No entanto, o tempo dedicado ao descanso aumentou com a idade, e o comportamento foi mais ativo nos machos subadultos e jovens em comparação ao de fêmeas e machos adultos (Koch e BiccaMarques, 2005). Portanto, os menores valores obtidos no VG e na PTP dos animais jovens (Tab. 2) podem ser justificados, considerando as maiores necessidades nutricionais da fase de crescimento, as atividades energeticamente mais dispendiosas nos mais jovens, embora não exista diferença na composição da dieta.

Tabela 2. Média e desvio-padrão das variáveis hematológicas e da concentração de proteína total plasmática de 36 adultos e jovens da espécie Alouatta caraya de vida livre, da região do Alto Rio Paraná, sul do Brasil, 2007

\begin{tabular}{lccc}
\hline Variável & Adulto $(\mathrm{n}=23)$ & Jovem $(\mathrm{n}=13)$ & $\mathrm{P}$ \\
\hline Hemácias $\left(\times 10^{6} / \mu \mathrm{L}\right)$ & $4,15 \pm 1,39$ & $3,67 \pm 0,73$ & $\mathrm{~ns}$ \\
Hemoglobina $(\mathrm{g} / \mathrm{dL})$ & $10,66 \pm 3,29$ & $10,22 \pm 3,40$ & $\mathrm{~ns}$ \\
Volume globular $(\%)$ & $39,46 \pm 3,53$ & $36,69 \pm 3,54$ & $<0,05$ \\
VGM (fL) & $107,21 \pm 46,07$ & $104,40 \pm 27,71$ & $\mathrm{~ns}$ \\
HGM (pg) & $27,44 \pm 10,08$ & $29,18 \pm 8,62$ & $\mathrm{~ns}$ \\
CHGM $(\%)$ & $26,91 \pm 7,48$ & $27,69 \pm 8,62$ & $\mathrm{~ns}$ \\
Leucócitos $\left(\times 10^{3} / \mu \mathrm{L}\right)$ & $11,33 \pm 7,47$ & $11,21 \pm 6,84$ & $\mathrm{~ns}$ \\
Segmentados $\left(\mathrm{x} 10^{3} / \mu \mathrm{L}\right)$ & $7,37 \pm 5,76$ & $6,98 \pm 6,10$ & $\mathrm{~ns}$ \\
Eosinófilos $\left(\mathrm{x} 10^{3} / \mu \mathrm{L}\right)$ & $0,60 \pm 0,78$ & $0,48 \pm 0,45$ & $\mathrm{~ns}$ \\
Linfócitos $\left(\mathrm{x} 10^{3} / \mu \mathrm{L}\right)$ & $3,22 \pm 1,87$ & $3,66 \pm 1,70$ & $\mathrm{~ns}$ \\
PTP $(\mathrm{g} / \mathrm{dl})$ & $7,91 \pm 0,53$ & $7,40 \pm 0,63$ & $<0,05$ \\
\hline VGM & & $g 03$ & \\
\hline
\end{tabular}

VGM: volume globular médio; HGM: hemoglobina globular média; CHGM: concentração de hemoglobina globular média; PTP: proteínas totais plasmáticas.

A condição ambiental de degradação do habitat e a pressão demográfica dos animais capturados em áreas de inundação para construção de hidrelétricas (Vie et al. 1998; Satake, 2006) podem ter influenciado algumas variáveis do eritrograma, uma vez que se observaram valores mais baixos de VG, VGM e HGM em $A$. seniculus (Vie et al., 1998) e hemácias e VG em A. caraya (Satake, 2006), em comparação com os resultados hematológicos obtidos para $A$. caraya neste estudo, e por Porter Júnior (1971) para $A$. villosa.

O número total de leucócitos obtido está próximo ao observado por Porter Júnior (1971) e é pouco maior do que o apontado em $A$. seniculus (Vie et al., 1998). Os números de leucócitos citados por Satake (2006) são mais elevados que os deste estudo, possivelmente devido à condição de ambiente e de captura.

O estresse é um estímulo adaptativo do organismo com respostas para lutar contra a mudança ambiental (Fowler, 1978) e apresenta um efeito distinto na função imunológica que, sob condições de estresse agudo, como captura, transporte e manipulação, promove imunoestimulação. Contudo, em situações de estresse crônico, como a degradação do habitat e a rarefação dos recursos naturais, ocorre imunodepressão (Vie et al., 1998; Dhabhar, 2002). Esse fato pode explicar a diferença entre os valores de leucócitos das espécies $A$. villosa e $A$. caraya em relação aos de $A$. seniculus (Vie et al., 1998) e $A$. caraya (Satake, 2006).

É possível que o medo da captura e da aproximação do homem e a resultante liberação de adrenalina tenham influenciado os resultados do leucograma, principalmente de neutrófilos, uma vez que este número tende a aumentar durante situações de estresse agudo, como observado em humanos (Isowa et al., 2004), em macaco Rhesus (Morrow-Tesch et al., 1993), em macaco-de-cheiro (Coe e Hall, 1996) e em sagui comum (Cunha et al., 2005). Os leucócitos parecem permanecer elevados até a cessação do 
estímulo de estresse agudo como forma de aumentar as defesas do organismo, recrutando neutrófilos para a circulação central e redistribuindo linfócitos para tecidos periféricos (Dhabhar, 2002).

Outro fator a ser considerado na interpretação dos resultados hematológicos é a anestesia. Os valores hematológicos obtidos de animais anestesiados podem ser considerados padrões, uma vez que dificilmente serão obtidos sem o efeito desses fármacos. Estudos com animais de cativeiro frequentemente utilizam a quetamina como anestésico (Larsson et al., 1999; Riviello e Wirz, 2001), entretanto, neste estudo os animais foram anestesiados com cloridratos de tiletamina e de zolazepam (TZ). O período de anestesia dos animais que recebem $\mathrm{TZ}$ é mais prolongado, mas os animais recobraram a consciência mais rapidamente, fato que torna este agente mais favorável, uma vez que os animais seriam recolocados no local da captura e soltos no meio ambiente. Além disso, a dose de TZ é inferior em relação à de quetamina, diminuindo o volume a ser injetado (Lee et al., 2003).

O uso da quetamina como agente anestésico proporciona diminuição nos valores do eritrograma, no número total de leucócitos e de linfócitos em macaco Rhesus, o que pode ser devido à reversão dos estímulos estressores, ou seja, à diminuição dos níveis de catecolaminas (Bennet et al., 1992). A redução de hemácias e de linfócitos circulantes pode ser resultante da redistribuição dessas células do sangue circulante para o baço e para os tecidos periféricos (Loomis et al., 1980; Wall et al., 1985). A diminuição da liberação das catecolaminas induzida pela anestesia pode ser apontada como causa das alterações hematológicas ocasionadas pelo agente anestésico, tendo este, portanto, efeito indireto. Não existem relatos dos efeitos específicos de $\mathrm{TZ}$ sobre os valores hematológicos, entretanto, pode-se supor que sejam semelhantes aos já comprovados para a quetamina.

A determinação dos valores hematológicos para animais em condições de vida livre pode ser um importante auxílio para o diagnóstico de algumas doenças, principalmente, com conhecimento adequado de outros fatores que possam influenciar estes valores, como diferenças em decorrência do sexo, da idade, do estresse ou do agente anestésico.

De forma geral, os valores hematológicos são semelhantes aos relatados em condições semelhantes, podendo ser admitidos como de referência para primatas deste gênero em vida livre.

\section{REFERÊNCIAS BIBLIOGRÁFICAS}

AGUIAR, L.M. Os primatas do Corredor do Alto Rio Paraná (Região de Porto Rico, Estados do Paraná e Mato Grosso do Sul): ocorrência, georreferenciamento e parâmetros populacionais. 2006. 110f. Dissertação (Mestrado) - Universidade Federal do Paraná, Curitiba.

AGUIAR, L.M.; LUDWIG, G.; SVOBODA, W.K. et al. Use of traps to capture black and gold howlers (Alouatta caraya) on the islands of the Upper Parana River, Southern Brazil. Am. J. Primatol., v.69, p.241-247, 2006.

AGUIAR, L.M.; REIS, N.R.; LUDWIG G, et al. Dieta, área de vida, vocalizações e estimativas populacionais de Alouatta guariba em um remanescente florestal no Norte do Estado do Paraná. Neotrop. Primates, v.11, p.78-86, 2003

AZEVEDO, R.B.; BICCA-MARQUES, J.C. Posturas de alimentação e tipos de locomoção em bugios ruivos (Alouatta guariba clamitans) em ambiente natural. In: CONGRESSO BRASILEIRO DE PRIMATOLOGIA. 11., 2005. Porto Alegre. Anais... Porto Alegre: PUCRS, 2005. p.76.(Resumo).

BARGER, A.M. The complete blood cell count: a powerful diagnostic tool. Vet. Clin. North Am.: Small Anim. Pract., v.33, p.1207-1222, 2003.

BENNET, J.S.; GOSSET, K.A.; McCARTHY, M.P. et al. Effects of ketamine hydrochloride on serum biochemical and hematologic variables in Rhesus monkeys (Macaca mulatta). Vet. Clin. Pathol., v.21, p.15-18, 1992.

COE, C.L.; HALL, N.R. Psychological disturbance alters thymic and adrenal hormone secretion in parallel but independent manner. Psychoneuroendocrinology, v.21, p.237-247, 1996.

COLES, E.H. Patologia clínica veterinária. 3.ed. São Paulo: Editora Manole, 1984. 566p. 
CUNHA, M.S.; LOPES, D.R.; SOUSA, M.B.C. Variação na contagem de leucócitos em Callithrix jacchus (Linnaeus, 1758) submetidos a uma situação de estresse agudo. Rev. Bras. Zoocienc., v.7, p.217-229, 2005.

CURI, P.R. Metodologia e análise da pesquisa em ciências biológicas. Botucatu: Tipomic, 1997. 263p.

DE LEEUW, N.K.M.; LOWESTEIN, L. The effect of intramuscular iron therapy on hematological values in normal men and women. Can. Med. Assoc. J., v.95, p.554-557, 1966.

DHABHAR, F.S. Stress-induced augmentation of immune function: The role of stress hormones, leukocyte trafficking, and cytokines. Brain Behav. Immum., v.16, p.785-798, 2002.

FOWLER, M.E. Restraint and handling of wild and domestic animals. Ames: Iowa State University Press, 1978. 332p.

HAREWOOD, W.J.; GILLIN, A.; HENNESSY, A. et al. The effects of the menstrual cycle, pregnancy and early lactation on haematology and plasma biochemistry in the baboon (Papio hamadryas). J. Med. Primatol., v.29, p.415-420, 2000.

HILST, C.L.S.; SVOBODA, W.K.; SPOHR, K.A.H. et al. Estudo e adaptação de protocolo de sedação à base de tiletamina/zolazepam em primates não-humanos da espécie Alouatta caraya. In: CONGRESSO BRASILEIRO DA ANCLIVEPA, 27., 2006, Vitória. Anais... Vitória: Anclivepa, 2006. (Resumo).

HULL, B.P.; DOUG-DEEN, R.; BUTCHER, L. et al. Laboratory diagnosis of yellow fever infections in humans, Alouatta monkeys and Haemagogus mosquitoes in Trinidad, 1978-1980. In: STUDIES on the natural history of yellow fever in Trinidad, Port of Spain: Caribbean Epidemiology Center, 1991. p.45-52.

ISOWA, T.; OHIRA, H.; MURASHIMA, S. Reactivity of immune, endocrine and cardiovascular parameters to active and passive acute stress. Biol. Psychol., v.65, p.101-120, 2004.

JAIN, N.C. Essentials of veterinary hematology. Philadelphia: Lea \& Febiger, 1993. 417p.

KOCH, F.; BICCA-MARQUES, J.C. Padrão de atividade e dieta de Alouatta guariba clamitans: Uma análise sexo-etária. In: CONGRESSO
BRASILEIRO DE PRIMATOLOGIA, 11., 2005, Porto Alegre. Anais... Porto Alegre: PUCRS, 2005. p.116. (Resumo).

LARSSON, M.H.M.A.; BIRGEL, E.H.; BENESI, F.J. et al. Padrões hematológicos em Cebus apella anestesiados com quetamina. Braz. J. Vet. Res. Anim. Sci., v.36, p.131-135, 1999.

LEE, J.; HONG, S.; LEE, S. et al. Immobilization with ketamine $\mathrm{HCl}$ and tiletamine-zolazepam in cynomolgus monkeys. $J$. Vet. Sci., v.4, p.187-191, 2003.

LOOMIS, M.R.; HENRICKSON, R.V.; ANDERSON, J.H. Effects of ketamine hydrochloride on the hemogram of Rhesus monkeys (Macaca mulatta). Lab. Anim. Sci., v.30, p.851-853, 1980.

MOORE, D.M. Hematology of nonhuman primates. In: FELDMAN, B.F.; ZINKL, J.G.; JAIN, N.C. (Eds). Schalm's veterinary hematology. 5.ed. Philadelphia: Lippincott, Williams \& Wilkins, 2000. p.1133-1144.

MORROW-TESCH, J.L.; McGLONE， J.J.; NORMAN, R.L. Consequences of restraint stress on natural killer activity, behavior, and hormone levels in rhesus macaques (Macaca mulatta). Psychoneuroendocrinology, v.18, p.383-395, 1993.

PORTER JUNIOR, J.A. Hematologic values of the black spider monkey (Ateles fusciceps), red spider monkey (Ateles geoffroyi), white face monkey (Cebus capucinus), and black howler monkey (Alouatta villosa). Lab. Anim. Sci., v.21, p.426-433, 1971.

RIVIELLO, C.; WIRZ, A. Haematology and blood chemistry of Cebus apella in relation to sex and age. J. Med. Primatol., v.30, p.308-312, 2001.

SATAKE, F. Contituintes sanguineos de bugiospretos (Alouatta caraya) e macacos-prego (Cebus apella) capturados no resgate de fauna da Usina Hidrelétrica Engenheiro Sérgio Motta. 2006. 60f. Tese (Doutorado) - Faculdade de Medicina Veterinária e Zootecnia, Universidade Estadual Paulista, Jaboticabal, SP.

SILVEIRA, R.M.M.; CODENOTTI, T.L. Interações sociais e dieta do bugio-ruivo, Alouatta guariba clamitans, no Parque Estadual de Itapuã, Rio Grande do Sul, Brasil. Neotrop. Primates, v.9, p.15-19, 2001. 
SVOBODA, W.K.; MALANSKI, L.S.; SHIOZAWA, M.M. et al. Dados biométricos de Alouatta caraya de vida livre de ilhas do alto Rio Paraná, região do município de Porto Rico, PR. In: CONGRESSO BRASILEIRO DE ZOOLOGIA, 12., 2006, Londrina. Anais... Londrina: EDUEL, 2006. (Resumo).

THOISY, B.; MICHEL, J.C.; VOGEL, I. et al. A survey of hemoparasite infections in free-ranging mammals and reptile in French Guiana. $J$. Parasitol., v.86, p.1035-1040, 2000.

THOISY, B.; VOGEL, I.; REYNES, J.M. et al. Health evaluation of translocated free ranging primates in French Guiana. Am. J. Primatol., v.54, p.1-16, 2001.
THRALL, M.A. Hematology of common non domestic mammals. In: _. Veterinary hematology and clinical chemistry. Baltimore: Lippicott Williams \& Wilkins, 2004. p.221-224.

VIE, J.C.; MOREAU, B.; THOISY, B. et al. Hematology and serum biochemical values of free-ranging red howler monkeys (Alouatta seniculus) from Freench Guiana. J. Zoo Wildl. Med., v.29, p.142-149, 1998.

WALL, H.S.; WORTHAMN, C.; ELSE, J.G. Effects of ketamine anaesthesia, stress and repeated bleeding on the haematology of Vervet monkeys. Lab. Anim., v.19, p.138-144, 1985. 